\title{
Macrophage subtype predicts lymph node metastasis in oesophageal adenocarcinoma and promotes cancer cell invasion in vitro
}

\author{
Wenqing Cao*, ${ }^{\star}$, Jeffrey H Peters ${ }^{\star 2}$, Dylan Nieman ${ }^{2}$, Meenal Sharma ${ }^{3}$, Thomas Watson ${ }^{2}$ and JiangZhou Yu ${ }^{3}$ \\ ${ }^{1}$ Department of Pathology, NYU School of Medicine, 560 First Avenue, New York, NY, USA; ${ }^{2}$ Department of Surgery, University of \\ Rochester Medical Center, Rochester, NY, USA and ${ }^{3}$ Department of Pathology and Laboratory Medicine, University of Rochester \\ Medical Centre, 601 Elmwood Avenue, Box 626, Rochester, NY, USA
}

Background: Currently, there is a lack of ideal biomarkers for predicting nodal status in preoperative stage of oesophageal adenocarcinoma (EAC) to aid optimising therapeutic options. We studied the potential of applying subtype macrophages to predict lymph node metastasis and prognosis in EAC.

Material and Methods: Fifty-three EAC resection specimens were immunostained with CD68, CD40 (M1), and CD163 (M2). Lymphatic vessel density (LVD) was estimated with the staining of D2-40. Subsequently, we tested if M2d macrophage could promote EAC cell migration and invasion.

Results: In EAC without neoadjuvant treatment, an increase in M2-like macrophage was associated with poor patient survival, independent of the locations of macrophages in tumour. The M2/M1 ratio that represented the balance between M2- and M1-like macrophages was significantly higher in nodal-positive EACs than that in nodal-negative EACs, and inversely correlated with patient overall survival. The M2/M1 ratio was not related to LVD. EAC cell polarised THP1 cell into M2d-like macrophage, which promoted EAC cell migration and invasion. Neoadjuvant therapy appeared to diminish the correlation between the M2/M1 ratio and survival.

Conclusions: The ratio of M2/M1 macrophage may serve as a sensitive marker to predict lymph node metastasis and poor prognosis in EAC without neoadjuvant therapy. M2d macrophage may have important roles in EAC metastasis.

The incidence of oesophageal adenocarcinoma (EAC) has increased considerably in many western countries over the past several decades (Cook et al, 2009; Steevens et al, 2010). Despite the recent advances in chemotherapy and radiation therapy, patients with EAC continue to have poor prognosis with an overall survival (OS) rate of 15-25\% (Ward and Mrsny, 2009). Currently, early selection of appropriate treatment strategy based on accurate cancer staging is the key to improve patient survival. However, before surgical resection, accurate cancer staging information, in particular lymph node status, cannot be achieved by pathologists or radiologists. Therefore, the strategy for treatment is guided by the clinical stage (Pollard, 2004;
Coffelt et al, 2009; Shabo et al, 2009; Kim et al, 2009a; Kurahara et al, 2011). Notably, 33-78\% of clinical T1-T2N0 EACs have nodal involvement detected in the oesophagectomy resection specimens. (Sepesi et al, 2010; Stiles et al, 2011; Zhang et al, 2012). The intrinsic pitfalls in clinical diagnosis have significantly constrained the power of adopting appropriate therapeutic approaches to further improve EAC patient treatment outcome, sometimes leading to over- or under-treatment of EAC patients. A reliable marker to predict lymph node metastasis before treatment may significantly ameliorate EAC patient OS rate.

EAC initiation and progression are generally believed to be closely associated with chronic inflammation. The local 
microenvironment created by infiltrating immune cells and cytokines in chronic inflammation may have crucial roles in tumorigenesis and metastatic potential (Colleypriest et al, 2009; Nguyen et al, 2010). Macrophage, a major component of the immune cell reaction seen in both primary and secondary tumours, has numerous roles in tumour initiation, progression, and metastasis. Given the heterogeneous nature of macrophage, different subtypes of macrophage adapt and respond to a large variety of micro-environmental signals within tumours. These subpopulations of macrophage have different types of receptor expression as well as cytokine and chemokine production. Classically activated macrophages (M1, pro-inflammatory cells) have pro-inflammatory activity, and may promote tumour growth in early stages of tumour development, but later appear to inhibit tumour growth. Alternatively activated macrophages (M2, anti-inflammatory cells) are oriented to tissue repair and immunoregulation; they stimulate angiogenesis, enhance tumour cell migration and invasion, and increase tumour metastasis (Mantovani et al, 1992, 2002; Mosser and Edwards, 2008; Mantovani and Sica, 2010; Qian and Pollard, 2010). Evidence has indicated that macrophages in tumour seem to have different effects on a variety of tumour progression (Zhang et al, 2012). A concept that has been embedded in the macrophage balance hypothesis suggests that macrophage may have dual functions in cancer (Mantovani et al, 1992; Mantovani and Sica, 2010). M2 macrophage seems to be associated with metastasis and poor prognosis in pancreas (Kurahara et al, 2011), liver (Kong et al, 2013), colon (Edin et al, 2012), and lung (Ma et al, 2010) cancers. Thus, we postulate that an increase of M2 and decrease of M1 macrophage in the tumour microenvironment may contribute to promoting metastasis of EAC. Alteration of the balance between M2 and M1 macrophage may be a predictor for tumour metastatic potential.

In oesophageal squamous cell carcinoma, data on the correlation of macrophage with lymph node spread and survival are contradicted in different studies (Koide et al, 2004; Guo et al, 2007). A recent study suggested that M2 macrophage associated with poor survival and lymph node metastasis (Shigeoka et al, 2013). Very few studies have done on the roles of subtype macrophage in EAC progression and prognosis, even though the incidence of adenocarcinoma of the oesophagus is rising dramatically worldwide, whereas the incidence of squamous cell carcinoma is decreasing (Brown et al, 2008). Our study was, therefore, aimed to explore whether a certain macrophage subpopulation correlated with lymph node metastasis in EAC patients, and whether it was associated with patient OS. This study was also aimed to comparatively evaluate the prognostic value of macrophage subpopulation in untreated and pre-treated EAC patients. In addition, we tested if M2d-like macrophage promoted EAC cell migration and invasion in vitro.

\section{MATERIALS AND METHODS}

Patients' recruitment and tissue samples. The pathology database at the University of Rochester Medical Center (URMC) from 2000 to 2011 was searched for oesophagectomy or gastroesophagectomy and EAC. A total of 53 resection specimens, including 33 cases without neoadjuvant therapy and 20 cases with neoadjuvant chemoradiation therapy were selected from a cohort of 258 EAC patients. The selection criteria were: (1) pathologic stage 1 to 3 tumour with or without lymph node metastasis; (2) at least 6 months' documented clinical follow-up; (3) availability of slides and tissue blocks. All selected cases were diagnosed microscopically on H\&E-stained slides and staged according to the seventh edition AJCC TNM (tumour/nodes/metastases) classification system. The diagnosis of adenocarcinoma for each case was confirmed by two GI pathologists (WC and MS). Clinical data were collected on gender, age at EAC, follow-up time, and survival. Other information including tumour size, stage, and differentiation were collected from the pathology report. Human tissue specimens obtained and processed at URMC were collected under the protocol (RSRB000038873) approved by the University of Rochester Research Subjects Review Board (RSRB). The institutional review board RSRB approved this retrospective study and waived the need for consent. Patient records/information was anonymised and de-identified before analysis.

Immunohistochemical and immunofluorescence staining. CD163 (scavenger receptor) and CD206/CD204 (mannose receptor) are often used to identify M2 subtype macrophages in human tissue samples (Pechkovsky et al, 2010; Durafourt et al, 2012). Several markers including CD40 (Aron-Wisnewsky et al, 2009), CD80 (Cui et al, 2013), iNOS (Edin et al, 2012), and HLA-DR (Ma et al, 2010) have been applied to identify M1 subtype macrophages in different studies. In this study, CD68 combined with CD163 was employed to identify M2-like macrophage subtype, and CD68 with CD40 was indicated for M1-like macrophage subtype.

Archival, formalin-fixed, paraffin-embedded tissue blocks from selected patients were procured from the Department of Pathology and Laboratory Medicine at URMC. Serial sections were immunohistochemically stained with CD68, CD40, CD163, and D2-40 antibodies as previously described (Ren et al, 2013). Briefly, $4 \mu \mathrm{m}$ paraffin sections were deparaffinised and this was followed by antigen retrieval in EDTA buffer $\left(\mathrm{pH} \mathrm{9.0)}\right.$ at $98^{\circ} \mathrm{C}$ for $20 \mathrm{~min}$. The rest of the procedure was done in a Dako automated instrument (Dako North America Inc., Carpinteria, CA, USA). Endogenous peroxidase activity was blocked with 3\% hydrogen peroxide. The slides were incubated with antibodies against CD68 (Clone KP1, 1:500, Dako North America Inc., Carpinteria, CA, USA), CD40 (sc-59044, 1:100, Santa Cruz Biotechnology, Santa Cruz, CA, USA), CD163 (sc-20066, 1:100, Santa Cruz Biotechnology), and D2-40 (M3619, 1:500, Dako North America Inc.) at room temperature for $1 \mathrm{~h}$ and were then incubated for $30 \mathrm{~min}$ with EnVision + System horseradish peroxidase-labelled polymer conjugated with biotinylated anti-mouse secondary antibody and 3,3'-diaminobenzidine substrate. All sections were counterstained with Mayer's haematoxylin.

Double immunofluorescence of CD40/CD68, CD163/CD68, and CD40/CD163 were performed using sequential method as reported (Pantano et al, 2013). In brief, $4 \mu \mathrm{m}$ paraffin sections were deparaffinised, rehydrated, and blocked with normal goat serum. The slides were then incubated with first primary antibody (CD40 or CD163) at room temperature for $1 \mathrm{~h}$, and then incubated with first secondary antibody (FITC-conjugated goat anti-mouse) for $30 \mathrm{~min}$ at room temperature. The slides were then washed with PBS Tween 20, and followed by blocking with second normal goat serum. Second primary (CD68 or CD163) and secondary antibodies (Texas red conjugated goat anti anti-mouse) were added as stated above. Immunofluorescence slides were counterstained with DAPI and covered with anti-fade mounting medium. Double immunohistochemistry of CD40/CD163 was also performed with Envision G/2 doublestain system (DAB + /permanent red) according to the manufactory's instructions.

Normal lymph node and colonic adenocarcinoma tissues were used as the positive controls. Negative controls were established by the replacement of primary antibodies with normal serum.

Evaluation of immunohistochemical and immunofluorescence staining. Tumour-infiltrating macrophages identified by the appropriate markers are defined as mononuclear cells that had oval to round nucleus and moderate to abundant cytoplasm, and 
showed strong membranous/cytoplasmic staining but not nuclear staining. The locations of macrophage seem to have different relationship with tumour progression and prognosis. Kong et al (2013) reported that in hepatocellular carcinoma, CD163-positive macrophage in peritumoral tissues was associated with poor OS, whereas CD163-positive cells in tumour centre had no prognostic value. In our study, we evaluated macrophage $\left(\mathrm{CD}^{+} 8^{+}\right)$, M1-like, and M2-like subtypes of macrophage in tumour centre and at tumour edge of EAC. Counting subtype macrophage was conducted as that described by a published report with modification (Kurahara et al, 2011). Briefly, after scanning the CD68 immunostained slide at low magnification $(\times 100)$, five areas with high macrophage density (CD68 staining hot spots) within the tumour and five areas at the tumour edge were selected. In the serial sections stained with CD40 or CD163, the corresponding areas of hot spots were identified. The numbers of M1-like $\left(\mathrm{CD} 40^{+}\right)$or M2-like $\left(\mathrm{CD} 63^{+}\right)$macrophages in each area were counted at high magnification $(\times 400)$ and the mean macrophage count (MC) for the tumour central area or tumour edge was calculated. The ratio of $\mathrm{M} 2 / \mathrm{M} 1$ was then calculated by dividing mean M2 count with mean M1 count for each case. Intratumoral and peritumoral lymphatic vessel density (LVD) was determined by calculating mean values of lymphatic vessels in five hot spots at high-power field $(\times 200)$ as reported (Saad et al, 2006).

Two pathologists counted macrophages with CD68, CD40, and CD163 staining, and lymphatic vessels with D2-40, independently. The counts from the two pathologists were considered 'agreement' when the differences between the counts of positive staining cells in the same case were less than $5 \%$. If the counts were different ( $>5 \%$ ), a consensus evaluation would be performed by the two pathologists to generate a consented count. The overall interobserval agreement (Kappa Score) for CD68, CD40, CD163 count, and D2-40 staining was $0.9,0.76,0.81$, and 0.88 , respectively.

Fluorescent images were obtained using an inverted microscope equipped for fluorescent microscopy. All images shown were obtained using oil immersion with a $\times 60$ objective lens.

Cell culture and THP1 cell polarisation. THP1 and SKGT EAC cell lines were obtained from the American Type Culture Collection. THP1 monocytes were cultured in RPM1 1640 containing $2 \mathrm{~mm}$ glutamine, $10 \mathrm{~mm}$ HEPES, $1 \mathrm{~mm}$ sodium pyruvate, $4.5 \mathrm{gl}^{-1}$ of glucose, $10 \%$ fetal calf serum, and $0.05 \mathrm{~mm}$ $\beta$-mercaptoethanol. SKGT cells were grown in RPMI 1640 supplemented with $2 \%$ fetal calf serum, penicillin, and gentamicin. Cells were cultured in $100 \mathrm{~mm}$ cell culture dishes or 6/24-well cell culture plates. $12 \mathrm{~h}$ before the beginning of each experiment, cells were cultured in serum-free medium with antibiotics. To polarise THP1 with SKGT cell, a co-culture was done with Transwell cell culture plate (Corning Life Sciences, Corning, NY, USA). The insert with $0.4 \mu \mathrm{m}$ pores was seeded with SKGT cells at $3 \times 10^{6}$ as induced group and without any cells as control group. THP1 cells were plated in the bottom chamber at $2 \times 10^{6}$. After 3 days culture, the insert was discarded, and the polarised THP1 cell was examined by microscopy and then used for RNA isolation or migration and invasion tests.

RNA isolation and RT-Q-PCR. Total RNA from the monocytes/ macrophage in 24-well cell culture plate was isolated using TRIZOL (Life Technologies, Grand Island, NY, USA) following manufacturer's instructions. One microgram of RNA was retrotranscribed to cDNA with reverse transcriptase (Invitrogen, Grand Island, NY, USA) and random hexamer primers. Cytokine/ chemokine mRNA expressions were analysed in duplicate by quantitative real-time PCR (Bio-Rad, Hercules, CA, USA) by corresponding primers. The relative expression of each gene was quantified by the comparative cycle threshold $\left(C_{\mathrm{t}}\right)$ method $\left(\Delta \Delta C_{\mathrm{t}}\right)$ by using GAPDH as the endogenous control.
Migration and invasion assays. After polarisation of THP1 cells, the insert containing SKGT cells was discarded. For migration test, a new insert with $8.0 \mu \mathrm{m}$ pores polyester membrane (Corning) was seeded with $2 \times 10^{5}$ SKGT Cells. To measure cell invasion, insert with $8.0 \mu \mathrm{m}$ pores was coated with Matrigel (BD Biosciences, San Jose, CA, USA) and $5 \times 10^{5} \mathrm{SKGT}$ cells were seeded. After 3 days co-culture, SKGT cells remained in the upper compartment were removed by cotton swabs, and those had invaded through the matrix were stained by cell stain $0.1 \%$ crystal violet and counted under a light microscope with five individual fields per insert. Results are presented as an average of triplicate experiments.

Statistical analyses. Statistical analyses were performed using the Prism 5 statistical package from GraphPad Software, Inc. (La Jolla, CA, USA). Overall survival was defined as the time from the date of diagnosis to the date of death or final clinical follow-up. The Kaplan-Meier method was used to estimate survival probabilities in patient subgroups, and the log-rank test for statistical comparisons. The correlation between $\mathrm{MC}, \mathrm{M} 2 / \mathrm{M} 1$ ratio, and clinicopathological characteristics was assessed using Student's $t$-test, $\chi^{2}$ test or Pearson's test. A $P$ value less than 0.05 was considered statistically significant.

\section{RESULTS}

A similar count of M1-like or M2-like macrophage was found in nodal-positive and -negative EAC without neoadjuvant therapy. To assure the specificity of the makers we chose for this study, immunofluorescence double staining (CD68/CD40 and CD68/CD163) was performed and showed that most CD40- or CD163-positive cells co-expressed CD68 (Supplementary Figure 1A). In addition, double immunofluorescence or immunohistochemical staining for CD40 and CD163 was conducted and demonstrated only rare cells expressed both CD40 and CD163 (Supplementary Figures $1 \mathrm{~A}$ and $1 \mathrm{~B}$ ). The results suggested that the markers we used in the study for identifying M1-like and M2-like subtypes of macrophage should be feasible and reliable in EAC specimens. Compared with EACs without lymph node metastasis, M1- like or M2-like macrophage, either located in tumour centre or at tumour edge, was not increased in EACs with positive lymph node(s) (Figure 1A and Supplementary Figure 2). Nevertheless, M2-like but not M1-like macrophage was significantly associated with poor patient OS (Figure 1B). $\mathrm{CD}^{+} 8^{+} \mathrm{MC}$ did not show any difference between EACs with or without nodal spread in either location (Supplementary Figure 3A). $\mathrm{CD}^{+} 8^{+}$macrophage did not relate to patient prognosis (Supplementary Figure 3B).

The M2/M1 ratio was significantly increased in nodal-positive EACs, and high M2/M1 ratio correlated with lymph node metastasis and poor OS. In this study, the ratio of $\mathrm{M} 2 / \mathrm{M} 1$ macrophage was significantly increased in EACs with nodal spread independent of locations, either in tumour centre (from $0.455 \pm 0.051$ to $1.417 \pm 0.325$ ) or at tumour edge (from $0.5918 \pm 0.155$ to $2.404 \pm 0.635$; Figure $2 \mathrm{~A}$ ). The median value of $\mathrm{M} 2 / \mathrm{M} 1$ ratio was 0.6 at tumour edge and 0.47 in tumour centre. Subsequently, the data indicated that a higher M2/M1 ratio at tumour edge $(>0.6)$ or in tumour centre $(>0.47)$ was strongly associated with lymph node metastasis (Figure 2B). Furthermore, a higher M2/M1 ratio was associated with poor prognosis of EAC patients (Figure 2C), also in a manner of independent of locations of macrophage in tumour. Notably, in our cohort, lymph node metastasis was related to poor prognosis (Supplementary Figure 4), which was in agreement with previous studies (Bollschweiler et al, 2006; Wijnhoven et al, 2007).

Associations of M2/M1 ratio with clinicopathological characteristics. In the 33 patients without neoadjuvant treatment, the 

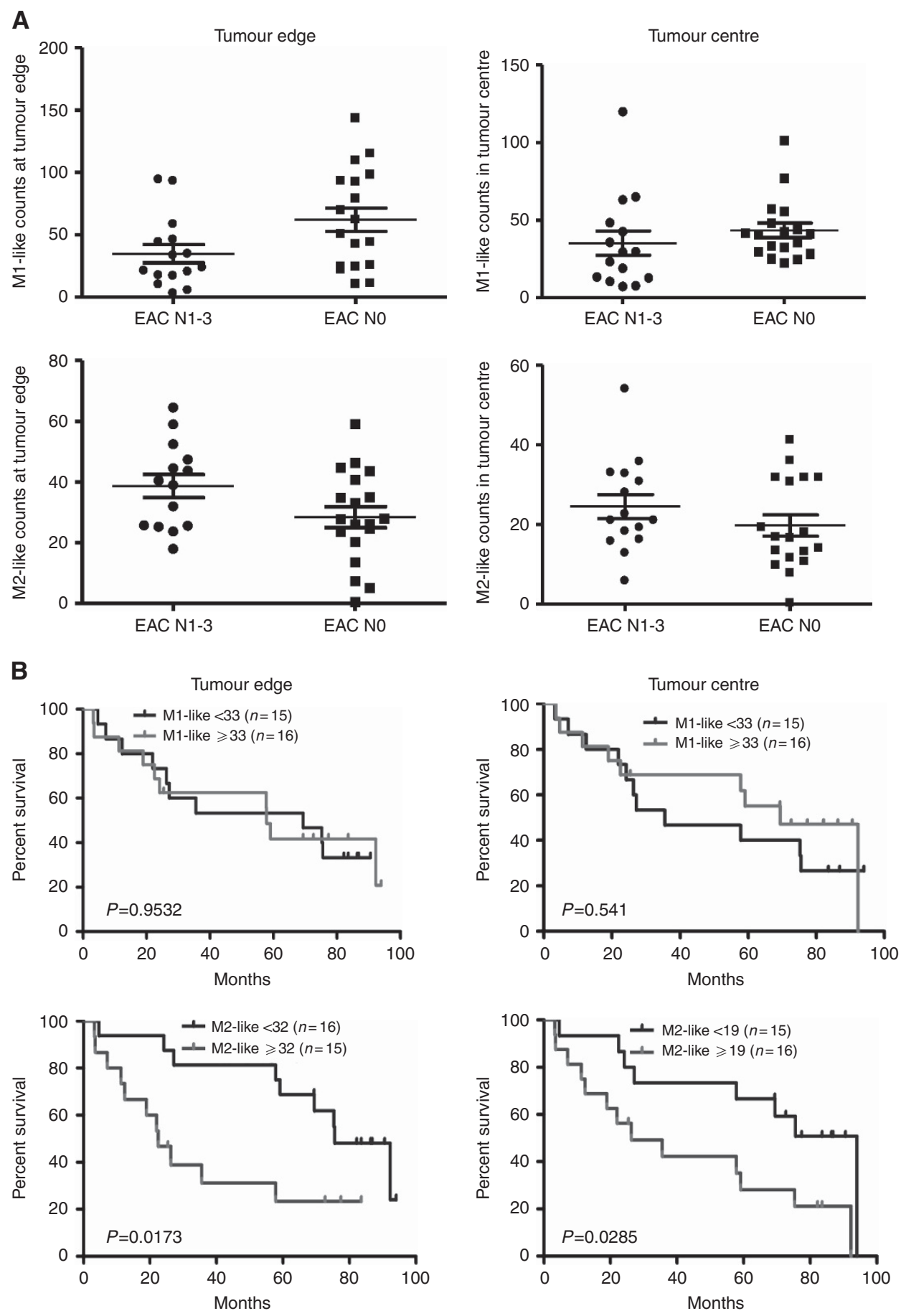

Figure 1. M2-like macrophage is associated with poor overall survival, but not associated with lymph node spread in EAC patients without neoadjuvant therapy. (A) The count of M1 or M2 macrophage in tumour centre and at tumour edge is not significantly different between EACs with (EAC N1-3) and without lymph node metastasis (EAC N0). (B) Kaplan-Meier overall survival curves of 31 EAC patients stratified by the counts of M1-like and M2-like macrophage based on the median number.

median age at the diagnosis of EAC was 68 years (range $=40-81$ years). About $88 \%$ of the patients were males. The ratio of M2/M1 macrophage was not associated with age, gender, tumour stage, or differentiation (Table 1). In the 20 patients who had chemo- or chemoradiation treatment before resection, 19 patients were males, only 1 patient was female. The age at the diagnosis of EAC ranged from 19 to 76 years with a median age of 61 years. The ratio of M2/ M1 macrophage did not correlate with any clinicopathological characteristics listed in Table 1.
Lymphatic vessel density was not associated with the ratio of M2/M1 or patient OS. To understand whether LVD correlated with lymph node metastasis, the ratio of $\mathrm{M} 2 / \mathrm{M} 1$ or patient survival in our study, D2-40 immunohistochemistry was performed (Supplementary Figure 5A). The median LVD was 4.8 lymphatic vessels per high-power field in tumour centre and 11.8 lymphatic vessels at tumour edge. LVD in tumour centre or at tumour edge seemed not to associate with the ratio of $\mathrm{M} 2 / \mathrm{M} 1$ macrophage or patient OS (Figure $3 \mathrm{~A}$ and $\mathrm{B}$ ). LVD in tumour centre was associated 

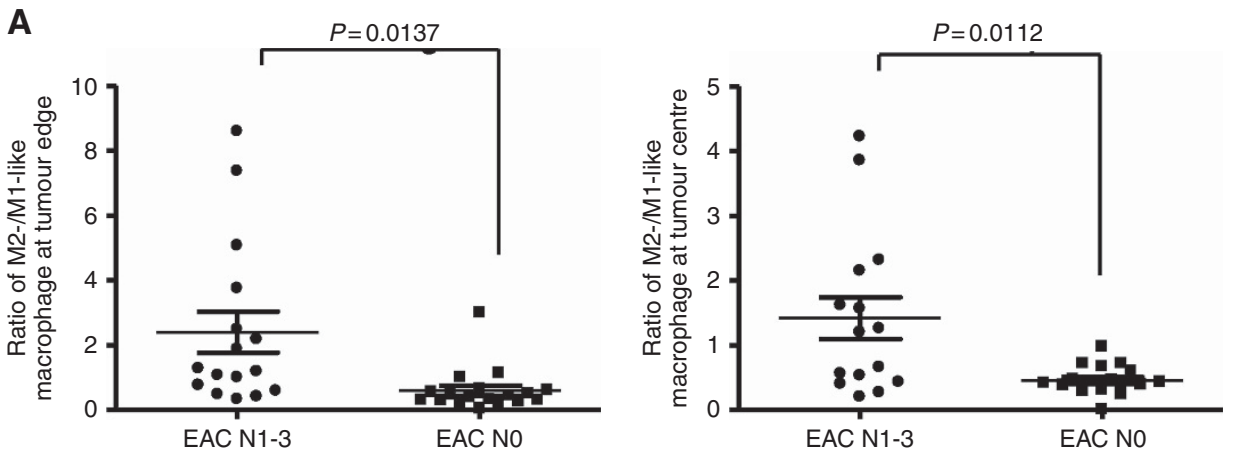

B

\begin{tabular}{|l|l|l|}
\hline $\begin{array}{l}\text { Ratio of M2-/M1- } \\
\text { like macrophage at } \\
\text { tumour edge }\end{array}$ & EAC N1-3 & EAC N0 \\
\hline$\geqslant 0.6$ & 10 & 5 \\
\hline$<0.6$ & 3 & 13 \\
\hline$P=0.0045$
\end{tabular}

\begin{tabular}{|l|l|l|}
\hline $\begin{array}{l}\text { Ratio of M2-/M1- } \\
\text { like macrophage in } \\
\text { tumour centre }\end{array}$ & EAC N1-3 & EAC N0 \\
\hline$\geqslant 0.47$ & 9 & 4 \\
\hline$<0.47$ & 5 & 13 \\
\hline
\end{tabular}

C
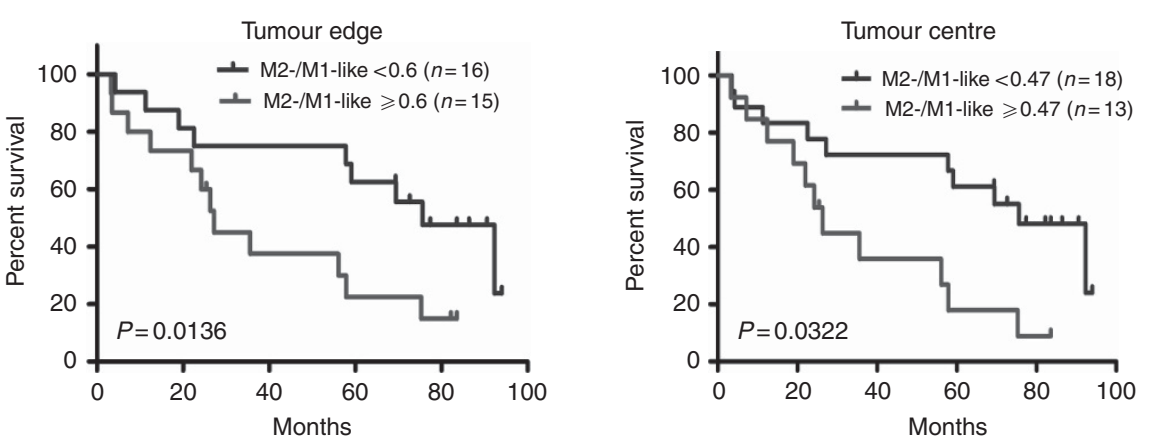

Figure 2. The associations of the ratio of M2/M1 macrophage in tumour centre or at tumour edge with nodal status and overall survival. (A) The ratio of M2/M1 macrophage is significantly higher in EAC N1-3 compared with that in EAC N0. (B) The ratio of M2/M1 macrophage in tumour centre or at tumour edge is related to lymph node metastasis ( $X^{2}$ test). (C) Kaplan-Meier overall survival curves of 31 EAC patients stratified by the ratio of M2/M1 macrophage in tumour centre and at tumour edge based on the median number.

with nodal spread (Supplementary Figure 5B). But we did not observe the association between LVD at tumour edge and nodal spread (Supplementary Figure 5B). The data implied that LVD may not be an ideal predictor for lymph node metastasis or prognosis in EAC.

M2d-like macrophage induced by EAC cell promoted cancer cell migration and invasion. To explore the potential mechanisms underlying the correlation of M2 macrophage with nodal spread, in this study, we tested if EAC cells could polarise monocytes into M2-like macrophages, and then evaluated if polarised macrophages promoted migration and invasion of EAC cells. After co-culture of EAC cell line, SKGT cells, with THP1 monocytes for 3 days, macrophage morphology was clearly seen (Figure 4A). The expression profile of chemokines/cytokines was used to characterise subtype macrophage, which included CD11b, IL-10, IL-12, CCL17, CCL18, CD206, and CD163. Compared with untreated THP1 cells, expressions of IL-10, CD163, CD206, and CCL18 were increased in SKGT-induced THP1 cells (Figure 4B), suggesting polarised THP1 cells displayed M2-like phenotype. High expression of CCL18 indicated the macrophage subtype was close to recently reported M2d-like phenotype (Wang et al, 2010). Subsequently, we continued to employ the co-culture system to investigate if M2d-like macrophage induced by SKGT cells promoted EAC cell migration and invasion. The data showed that
M2d-like macrophage not only stimulated EAC cell migration, but also increased cancer cell invasion (Figures 4C and D).

Neoadjuvant therapy diminished the correlation between the ratio of $\mathrm{M} 2 / \mathrm{M1}$ macrophage and lymph node metastasis or patient survival. Twenty EAC patients with neoadjuvant therapy were included in this study. The data indicated that the counts of M1-like and M2-like macrophage in tumour centre or at tumour edge with and without nodal spread was not statistically different in EACs with neoadjuvant treatment (Supplementary Figure 6). The ratio of $\mathrm{M} 2 / \mathrm{M} 1$ macrophage in tumour centre or at tumour edge after neoadjuvant therapy was not associated with lymph node metastasis or OS (Figures $5 \mathrm{~A}$ and $\mathrm{B}$ ).

\section{DISCUSSION}

In this study, we revealed that the ratio of M2/M1 macrophage in tumour centre or at tumour edge of EAC was strongly associated with nodal spread and poor OS, suggesting that a relative increase of M2-like macrophage may be a potential marker for predicting lymph node metastasis in EAC biopsy specimens without neoadjuvant therapy. Furthermore, the in vitro studies indicated that polarised THP1 cell by EAC cell might be M2d-like macrophage based on cytokine expression profile, which could 
Table 1. The associations of the ratio of M2/M1 macrophage and clinicopathological characteristics in EAC patients without or with neoadjuvant therapy

\begin{tabular}{|c|c|c|c|c|}
\hline & \multicolumn{2}{|c|}{ Tumour centre } & \multicolumn{2}{|c|}{ Tumour edge } \\
\hline & Mean \pm s.d. & $P$ & Mean \pm s.d. & $P$ \\
\hline \multicolumn{5}{|c|}{ Without neoadjuvant treatment } \\
\hline $\begin{array}{l}\text { Age (years) } \\
\qquad 68(n=16) \\
>68(n=17)\end{array}$ & $\begin{array}{l}0.90 \pm 1.30 \\
0.79 \pm 0.67\end{array}$ & 0.77 & $\begin{array}{l}1.78 \pm 2.61 \\
1.16 \pm 1.28\end{array}$ & 0.73 \\
\hline $\begin{array}{l}\text { Gender } \\
\quad F(n=4) \\
M(n=29)\end{array}$ & $\begin{array}{l}0.19 \pm 0.13 \\
0.94 \pm 1.03\end{array}$ & 0.16 & $\begin{array}{l}0.44 \pm 0.20 \\
1.61 \pm 2.12\end{array}$ & 0.27 \\
\hline $\begin{array}{l}\text { Tumour size }(\mathrm{cm}) \\
\quad \leqslant 2.6(n=15) \\
>2.6(n=18)\end{array}$ & $\begin{array}{l}0.76 \pm 1.02 \\
0.92 \pm 1.01 \\
\end{array}$ & 0.64 & $\begin{array}{l}1.25 \pm 1.96 \\
1.65 \pm 0.75 \\
\end{array}$ & 0.36 \\
\hline $\begin{array}{l}\text { Tumour stage } \\
\text { T1 }(n=20) \\
\text { T2 }(n=8) \\
\text { T3 }(n=5)\end{array}$ & $\begin{array}{l}0.81 \pm 0.96 \\
0.77 \pm 1.27 \\
1.17 \pm 0.76\end{array}$ & 0.49 & $\begin{array}{l}1.45 \pm 2.24 \\
0.77 \pm 1.27 \\
1.39 \pm 1.50\end{array}$ & 0.10 \\
\hline $\begin{array}{l}\text { Tumour grade } \\
\text { Well }(n=7) \\
\text { Moderate }(n=16) \\
\text { Poor }(n=10)\end{array}$ & $\begin{array}{l}0.41 \pm 0.21 \\
1.06 \pm 1.23 \\
0.82 \pm 0.85\end{array}$ & 0.36 & $\begin{array}{l}0.86 \pm 1.03 \\
2.00 \pm 2.54 \\
1.06 \pm 1.47\end{array}$ & 0.37 \\
\hline \multicolumn{5}{|c|}{ With neoadjuvant treatment } \\
\hline $\begin{array}{l}\text { Age (years) } \\
\quad \leqslant 61(n=11) \\
\quad>61(n=9)\end{array}$ & $\begin{array}{l}0.90 \pm 0.52 \\
1.02 \pm 0.96\end{array}$ & 0.72 & $\begin{array}{l}1.03 \pm 1.00 \\
1.11 \pm 0.96\end{array}$ & 0.86 \\
\hline $\begin{array}{l}\text { Gender } \\
\quad F(n=1) \\
M(n=19) \\
\end{array}$ & & NA & & NA \\
\hline $\begin{array}{l}\text { Tumour size }(\mathrm{cm}) \\
\quad \leqslant 4.1(n=15) \\
>4.1(n=18)\end{array}$ & $\begin{array}{l}0.95 \pm 0.93 \\
0.96 \pm 0.51\end{array}$ & 0.98 & $\begin{array}{l}0.98 \pm 0.97 \\
1.11 \pm 1.02\end{array}$ & 0.77 \\
\hline $\begin{array}{l}\text { Tumour stage } \\
\text { T1-2 }(n=10) \\
\text { T3-4 }(n=10) \\
\end{array}$ & $\begin{array}{l}1.16 \pm 1.08 \\
0.82 \pm 0.36 \\
\end{array}$ & 0.31 & $\begin{array}{l}1.23 \pm 1.06 \\
0.96 \pm 0.95\end{array}$ & 0.54 \\
\hline $\begin{array}{l}\text { Tumour grade } \\
\text { Well/moderate }(n=8) \\
\text { Poor }(n=12)\end{array}$ & $\begin{array}{l}0.68 \pm 0.18 \\
1.07 \pm 0.85\end{array}$ & 0.28 & $\begin{array}{l}0.78 \pm 0.38 \\
1.19 \pm 1.12\end{array}$ & 0.41 \\
\hline $\begin{array}{l}\text { Abbreviations: } E A C=\text { oesop } \\
\text { applicable. }\end{array}$ & adenocarcin & & le; $M=$ male; & $=$ not \\
\hline
\end{tabular}

be the important subtype of macrophage contributes to nodal spread in EAC.

In oesophageal cancer, the studies on macrophage association with lymph node metastasis or poor prognosis were reported in squamous cell carcinoma (Koide et al, 2004; Guo et al, 2007). A recent study further indicated that M2-like macrophage was associated with poor disease-free survival (Shigeoka et al, 2013). In correlation with the reports, M2-like macrophage either in tumour centre or at edge of EAC in our study showed a correlation with poor OS. However, there is inconsistency in data on correlation between MC and lymph node metastasis in oesophageal squamous cell carcinoma (Ohashi et al, 2000; Noguchi et al, 2002; Shigeoka et al, 2013). The contradictive data may be interpreted by potential biases from sample size of the cohort or the different methods of counting macrophages. Nevertheless, the inconsistency may indicate that the count of macrophage is not a sensitive and reliable predictor for lymph node metastasis in oesophageal cancer. The count of macrophage $\left(\mathrm{CD} 8^{+}\right), \mathrm{M} 1$-like, or M2-like macrophage was not significantly different between EAC with and without nodal spread in this study providing evidence to support this notion.

Increasing evidence has suggested that the dynamic change of macrophage subtypes in tumour has important roles in tumour metastasis (Qian and Pollard, 2010; Horimoto et al, 2012). The ratio of M1 and M2 macrophage was reported as an independent predictor of survival in gastric cancer (Pantano et al, 2013). In this study, the M2/M1 ratio in tumour centre or at tumour edge was obviously increased in EACs with nodal spread, and strongly associated with lymph node metastasis and OS. These data suggest that the ratio of M2/M1 macrophage would be a more reliable predictor for lymph node metastasis and prognosis in EAC. In addition, our data indicated that the ratio of M2/M1 macrophage in tumour centre and at tumour edge had similar power to predict nodal status in EAC. This gives rise to a possibility that small human specimens like biopsy samples could be used to predict lymph node metastasis by evaluating the ratio of $\mathrm{M} 2 / \mathrm{M} 1$.

Macrophages are plastic cells and can be polarised into M1 and M2 subtypes on the basis of their ability to produce IL-12 and IL-10, respectively (Mantovani et al, 2002; Gordon and Taylor, 2005). M2 macrophage is further subdivided into M2a, M2b, M2c, and M2d based on cytokine/chemokine expression profiles (Duluc et al, 2007; Wang et al, 2010). Evidences have shown that the products from tumour cells can activate/polarise macrophages (Kim et al, 2009b; Sica and Mantovani, 2012; Stewart et al, 2012). In this study, after co-culture with EAC cell line SKGT, THP1 cells were polarised to M2-like macrophage displaying high expression of IL-10, CD206, and CD163, and low expression of IL-12 and CD11b. CCL18 expression was also greatly increased in SKGTinduced M2-like macrophage, suggesting the M2-like macrophage might be M2d subset (Duluc et al, 2007; Wang et al, 2010). Our study may be the first report to successfully induce M2d-like macrophage from THP1 cell using EAC cancer cell. The data from migration and invasion experiments demonstrated M2d-like macrophage stimulated EAC cell migration and invasion. The above findings were in agreement with a recent report in which M2d macrophage promoted tumour migration and invasion (Li et al, 2011). Notably, our data indicated that tumour LVD was not associated with lymph node metastasis or prognosis in EACs. These data suggested that M2d-like macrophage might have important roles in EAC metastasis through promoting tumour cell migration and invasion. If this is the case, developing an M2d-specific maker may be more efficient in predicting lymph node metastasis in EAC than the ratio of M2/M1 macrophage dose.

In the United States, preoperative chemoradiation therapy has been employed by many institutions as the standard of care for oesophageal cancer, although the debate about its overall effect on survival still exists (Campbell and Villaflor, 2010; Ajani et al, 2011). Chemoradiation therapy not only impacts the macrophage composition and function, but also regulates its anticancer effect in tumour (De Palma and Lewis, 2013). It is necessary to understand if the correlation between the ratio of M2/ M1 macrophage and nodal spread is impacted in EACs with neoadjuvant therapy. The experiments with EACs post neoadjuvant treatment showed that the ratio of $\mathrm{M} 2 / \mathrm{M} 1$ macrophage in tumour centre or at tumour edge was not associated with nodal spread and OS. The data indicated that neoadjuvant therapy altered the composition of macrophage in EAC. This suggested that patient's treatment history should be taken into account when selecting patients and interpreting data in future studies.

In conclusion, our study is the first one to evaluate the correlation of M1-like, M2-like macrophage, and the ratio of M2/M1 macrophage, with lymph node metastasis and OS in EAC. Our findings shed the light into the possibility of using the ratio of M2/M1 macrophage in biopsy samples to predict lymph node spread in EACs without neoadjuvant therapy. The in vitro data, for the first time, indicate that EAC cell can polarise monocyte into M2d-like phenotype, which promotes the migration and invasion of EAC cells, suggesting that M2d macrophage may have an 

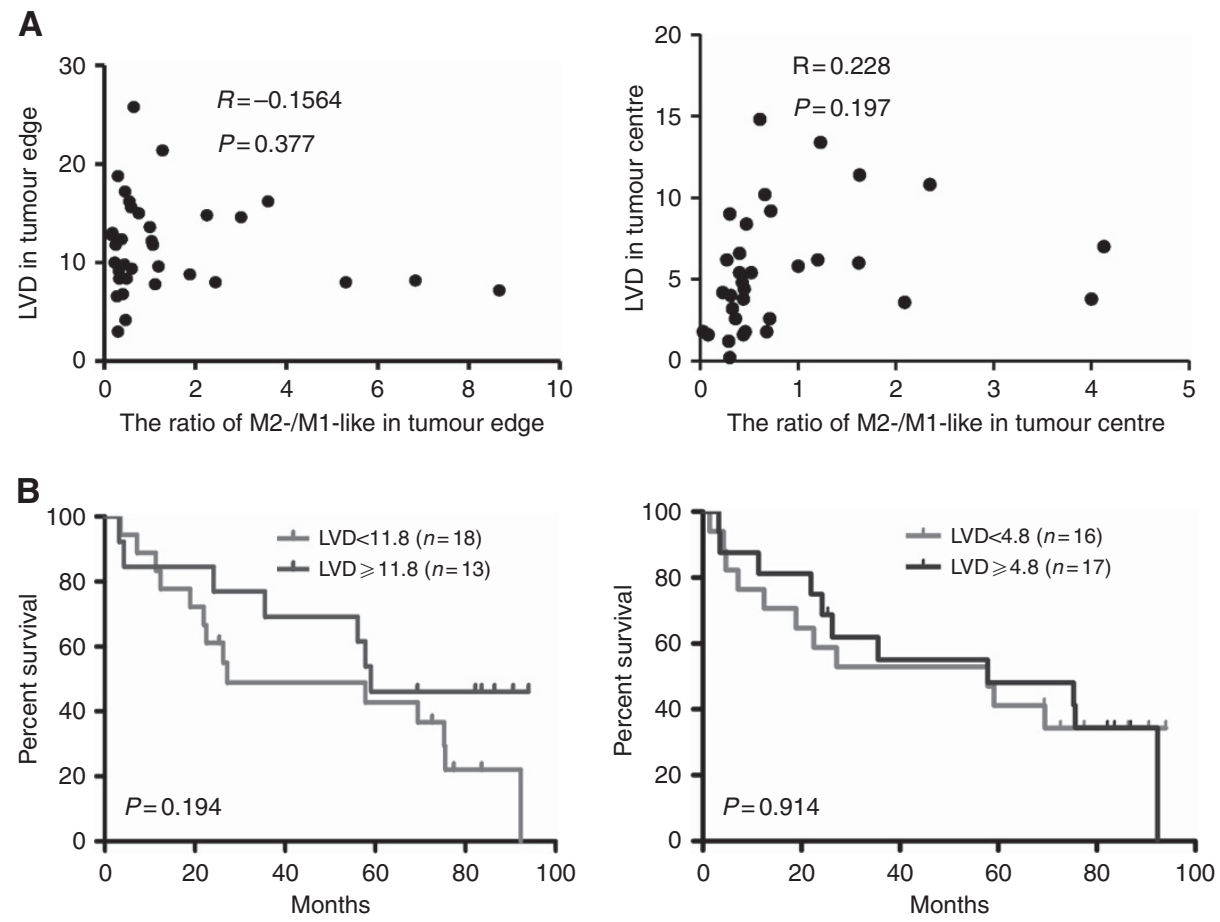

Figure 3. Lymphatic vessel density (LVD) in tumour centre or at tumour edge is not associated with the ratio of M2/M1 macrophage or patient overall survival. (A) Correlation of LVD in tumour centre or at tumour edge with the ratio of M2/M1 macrophage (Pearson's test). (B) Kaplan-Meier overall survival curves of 31 EAC patients stratified by LVD in tumour centre and at tumour edge based on the median number.

A

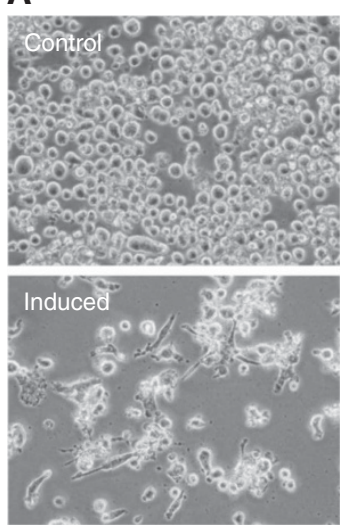

C

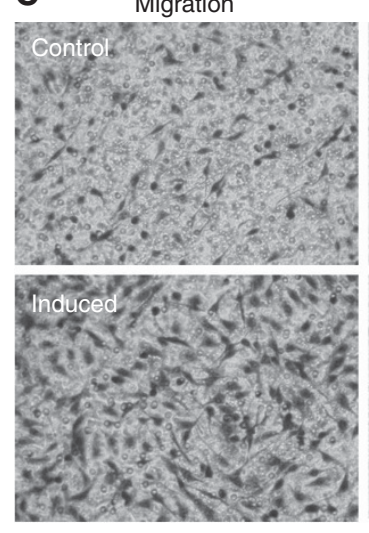

Invasion

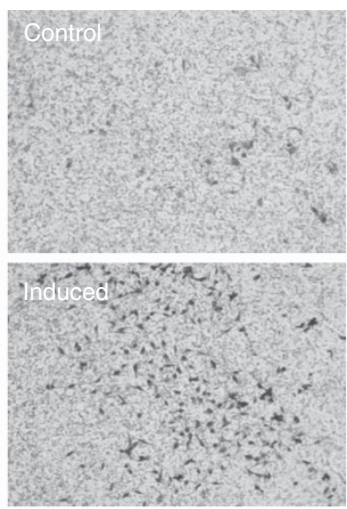

B

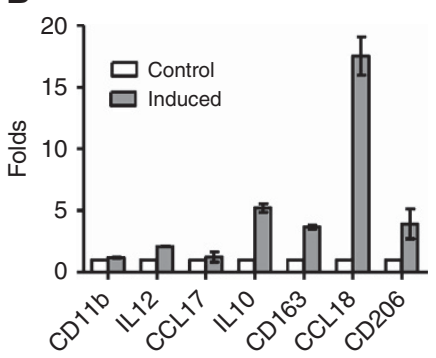

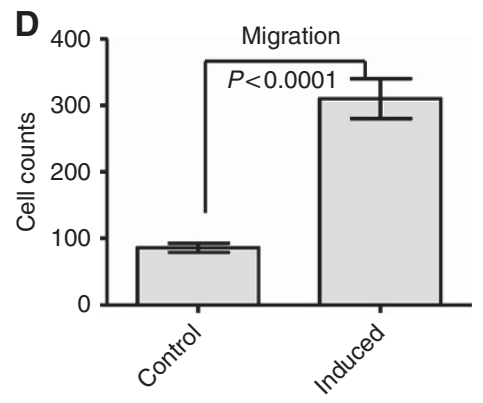

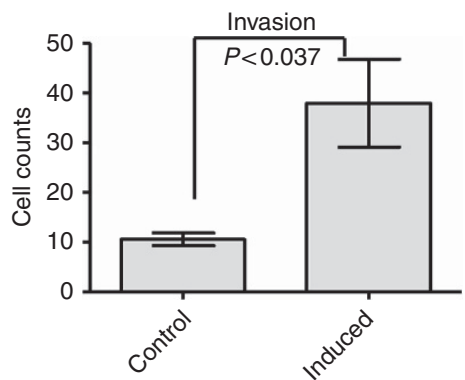

Figure 4. Induction of THP1 monocyte into M2-like macrophage with human EAC cell line SKGT. (A) Images of THP1 cells in control and polarised groups after co-cultured for 3 days without or with SKGT cells. (B) Quantification data from RT-Q-PCR show the expressions of cytokines/ chemokines ( $n=3$ individual experiments). (C) Representative images of EAC SKGT cells after migration and invasion. (D) Quantification data from migration and invasion experiments ( $n=5$ individual experiments). 

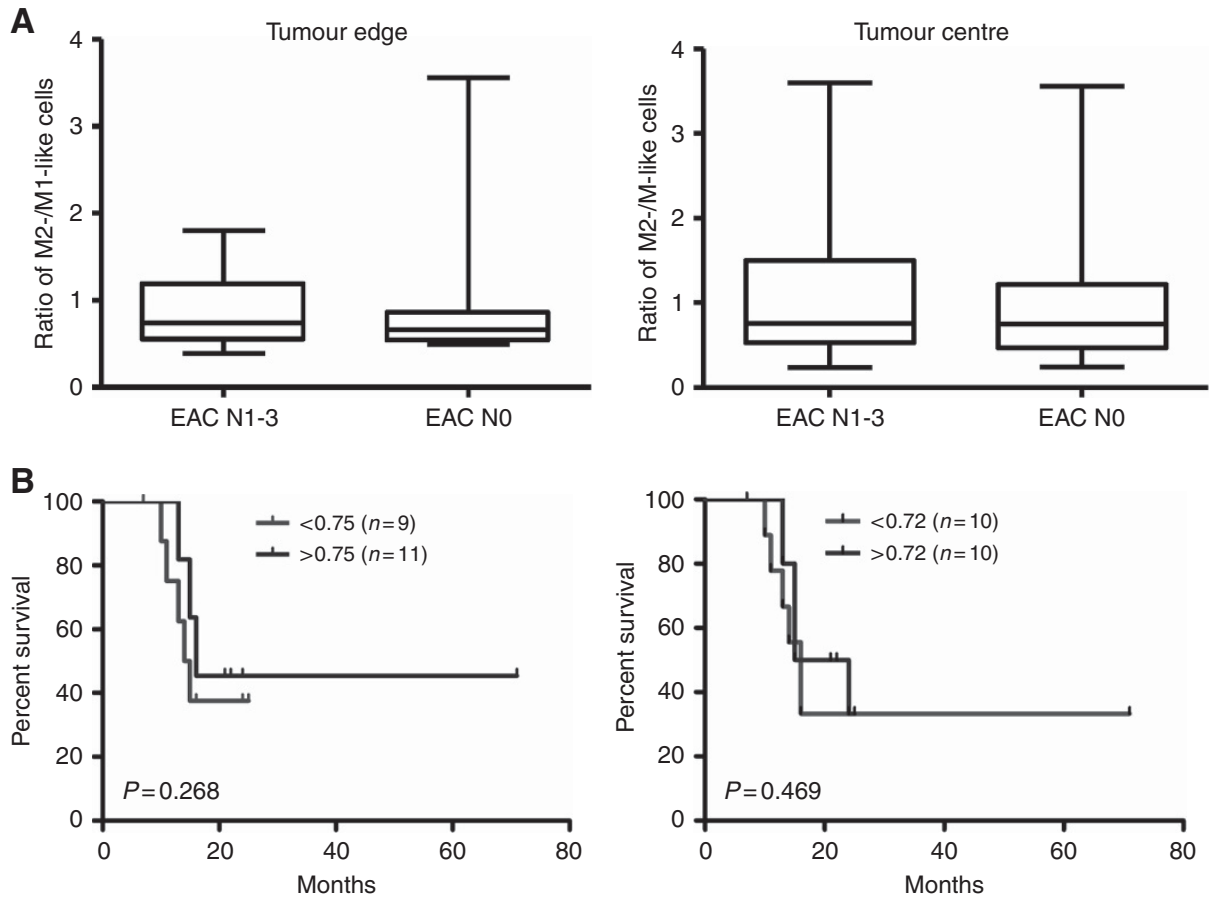

Figure 5. The associations of the ratio of M2/M1 macrophage with lymph node metastasis and overall survival in EACs with neoadjuvant therapy. (A) The ratio of M2/M1 macrophage is not increased in EAC N1-3 compared with that in EAC N0. (B) Kaplan-Meier overall survival curves of 20 EAC patients stratified by the ratio of M2/M1 macrophage in tumour centre and at tumour edge based on the median number.

important role in driving lymph node metastasis in EAC. More studies are needed to further reveal the role of M2d macrophage in EAC progression and metastasis.

\section{ACKNOWLEDGEMENTS}

We sincerely thank Ms Qi Yang for assistance in immunohistochemical studies, and Ms Liana Toia for assistance in cell culture studies and RT-PCR. The work was supported by funding from the Department of Pathology and Department of Surgery at University of Rochester Medical Centre. The project described in this publication was supported by the University of Rochester CTSA award number UL1 TR000042 from the National Centre for Advancing Translational Sciences of the National Institutes of Health.

\section{CONFLICT OF INTEREST}

The authors declare no conflict of interest.

\section{REFERENCES}

Ajani JA, Barthel JS, Bentrem DJ, D’Amico TA, Das P, Denlinger CS, Fuchs CS, Gerdes H, Glasgow RE, Hayman JA, Hofstetter WL, Ilson DH, Keswani RN, Kleinberg LR, Korn WM, Lockhart AC, Mulcahy MF, Orringer MB, Osarogiagbon RU, Posey JA, Sasson AR, Scott WJ, Shibata S, Strong VE, Varghese Jr TK, Warren G, Washington MK, Willett C, Wright CD. National Comprehensive Cancer Network (2011) Esophageal and esophagogastric junction cancers. J Natl Compr Canc Netw 9(8): 830-887.

Aron-Wisnewsky J, Tordjman J, Poitou C, Darakhshan F, Hugol D, Basdevant A, Aissat A, Guerre-Millo M, Clément K (2009) Human adipose tissue macrophages: $\mathrm{m} 1$ and $\mathrm{m} 2$ cell surface markers in subcutaneous and omental depots and after weight loss. J Clin Endocrinol Metab 94(11): 4619-4623.
Bollschweiler E, Baldus SE, Schroder W, Schneider PM, Holscher AH (2006) Staging of esophageal carcinoma: length of tumor and number of involved regional lymph nodes. Are these independent prognostic factors? J Surg Oncol 94(5): 355-363.

Brown LM, Devesa SS, Chow WH (2008) Incidence of adenocarcinoma of the esophagus among white Americans by sex, stage, and age. J Natl Cancer Inst 100(16): 1184-1187.

Campbell NP, Villaflor VM (2010) Neoadjuvant treatment of esophageal cancer. World J Gastroenterol 16(30): 3793-3803.

Coffelt SB, Hughes R, Lewis CE (2009) Tumor-associated macrophages: effectors of angiogenesis and tumor progression. Biochim Biophys Acta 1796(1): 11-18

Colleypriest BJ, Ward SG, Tosh D (2009) How does inflammation cause Barrett's metaplasia? Curr Opin Pharmacol 9(6): 721-726.

Cook MB, Chow WH, Devesa SS (2009) Oesophageal cancer incidence in the United States by race, sex, and histologic type, 1977-2005. Br J Cancer 101(5): 855-859.

Cui YL, Li HK, Zhou HY, Zhang T, Li Q (2013) Correlations of tumorassociated macrophage subtypes with liver metastases of colorectal cancer. Asian Pac J Cancer Prev 14(2): 1003-1007.

De Palma M, Lewis CE (2013) Macrophage regulation of tumor responses to anticancer therapies. Cancer Cell 23(3): 277-286.

Duluc D, Delneste Y, Tan F, Moles MP, Grimaud L, Lenoir J, Preisser L, Anegon I, Catala L, Ifrah N, Descamps P, Gamelin E, Gascan H, Hebbar M, Jeannin P (2007) Tumor-associated leukemia inhibitory factor and IL-6 skew monocyte differentiation into tumor-associated macrophage-like cells. Blood 110(13): 4319-4330.

Durafourt BA, Moore CS, Zammit DA, Johnson TA, Zaguia F, Guiot MC, Bar-Or A, Antel JP (2012) Comparison of polarization properties of human adult microglia and blood-derived macrophages. Glia 60(5): 717-727.

Edin S, Wikberg ML, Dahlin AM, Rutegard J, Oberg A, Oldenborg PA, Palmqvist R (2012) The distribution of macrophages with a M1 or M2 phenotype in relation to prognosis and the molecular characteristics of colorectal cancer. PLoS ONE 7(10): e47045.

Gordon S, Taylor PR (2005) Monocyte and macrophage heterogeneity. Nat Rev Immunol 5(12): 953-964.

Guo SJ, Lin DM, Li J, Liu RZ, Zhou CX, Wang DM, Ma WB, Zhang YH, Zhang SR (2007) Tumor-associated macrophages and CD3-zeta expression of tumor-infiltrating lymphocytes in human esophageal squamous-cell carcinoma. Dis Esophagus 20(2): 107-116. 
Horimoto Y, Polanska UM, Takahashi Y, Orimo A (2012) Emerging roles of the tumor-associated stroma in promoting tumor metastasis. Cell Adh Migr 6(3): 193-202.

Kim S, Takahashi H, Lin WW, Descargues P, Grivennikov S, Kim Y, Luo JL, Karin M (2009a) Carcinoma-produced factors activate myeloid cells through TLR2 to stimulate metastasis. Nature 457(7225): 102-106.

Kim TJ, Kim HY, Lee KW, Kim MS (2009b) Multimodality assessment of esophageal cancer: preoperative staging and monitoring of response to therapy. Radiographics 29(2): 403-421.

Koide N, Nishio A, Sato T, Sugiyama A, Miyagawa S (2004) Significance of macrophage chemoattractant protein-1 expression and macrophage infiltration in squamous cell carcinoma of the esophagus. Am J Gastroenterol 99(9): 1667-1674.

Kong LQ, Zhu XD, Xu HX, Zhang JB, Lu L, Wang WQ, Zhang QB, Wu WZ, Wang L, Fan J, Tang ZY, Sun HC (2013) The clinical significance of the CD163 + and CD68 + macrophages in patients with hepatocellular carcinoma. PLoS ONE 8(3): e59771.

Kurahara H, Shinchi H, Mataki Y, Maemura K, Noma H, Kubo F, Sakoda M, Ueno S, Natsugoe S, Takao S (2011) Significance of M2-polarized tumorassociated macrophage in pancreatic cancer. J Surg Res 167(2): e211-e219.

Li Y, Cai L, Wang H, Wu P, Gu W, Chen Y, Hao H, Tang K, Yi P, Liu M, Miao S, Ye D (2011) Pleiotropic regulation of macrophage polarization and tumorigenesis by formyl peptide receptor-2. Oncogene 30(36): 3887-3899.

Ma J, Liu L, Che G, Yu N, Dai F, You Z (2010) The M1 form of tumorassociated macrophages in non-small cell lung cancer is positively associated with survival time. BMC Cancer 10: 112.

Mantovani A, Bottazzi B, Colotta F, Sozzani S, Ruco L (1992) The origin and function of tumor-associated macrophages. Immunol Today 13(7): 265-270.

Mantovani A, Sica A (2010) Macrophages, innate immunity and cancer: balance, tolerance, and diversity. Curr Opin Immunol 22(2): 231-237.

Mantovani A, Sozzani S, Locati M, Allavena P, Sica A (2002) Macrophage polarization: tumor-associated macrophages as a paradigm for polarized M2 mononuclear phagocytes. Trends Immunol 23(11): 549-555.

Mosser DM, Edwards JP (2008) Exploring the full spectrum of macrophage activation. Nat Rev Immunol 8(12): 958-969.

Nguyen GH, Schetter AJ, Chou DB, Bowman ED, Zhao R, Hawkes JE, Mathé EA, Kumamoto K, Zhao Y, Budhu A, Hagiwara N, Wang XW, Miyashita M, Casson AG, Harris CC (2010) Inflammatory and microRNA gene expression as prognostic classifier of Barrett's-associated esophageal adenocarcinoma. Clin Cancer Res 16(23): 5824-5834.

Noguchi T, Takeno S, Shibata T, Uchida Y, Yokoyama S, Muller W (2002) VEGF-C expression correlates with histological differentiation and metastasis in squamous cell carcinoma of the esophagus. Oncol Rep 9(5): 995-999.

Ohashi Y, Ishibashi S, Suzuki T, Shineha R, Moriya T, Satomi S, Sasano H (2000) Significance of tumor associated tissue eosinophilia and other inflammatory cell infiltrate in early esophageal squamous cell carcinoma. Anticancer Res 20(5A): 3025-3030.

Pantano F, Berti P, Guida FM, Perrone G, Vincenzi B, Amato MM, Righi D, Dell'aquila E, Graziano F, Catalano V, Caricato M, Rizzo S, Muda AO, Russo A, Tonini G, Santini D (2013) The role of macrophages polarization in predicting prognosis of radically resected gastric cancer patients. J Cell Mol Med 17(11): 1415-1421.

Pechkovsky DV, Prasse A, Kollert F, Engel KM, Dentler J, Luttmann W, Friedrich K, Müller-Quernheim J, Zissel G (2010) Alternatively activated alveolar macrophages in pulmonary fibrosis-mediator production and intracellular signal transduction. Clin Immunol 137(1): 89-101.

Pollard JW (2004) Tumour-educated macrophages promote tumour progression and metastasis. Nat Rev Cancer 4(1): 71-78.

Qian BZ, Pollard JW (2010) Macrophage diversity enhances tumor progression and metastasis. Cell 141(1): 39-51.

Ren B, Zakharov V, Yang Q, McMahon L, Yu J, Cao W (2013) MACC1 is related to colorectal cancer initiation and early-stage invasive growth. Am J Clin Pathol 140(5): 701-707.

Saad RS, Kordunsky L, Liu YL, Denning KL, Kandil HA, Silverman JF (2006) Lymphatic microvessel density as prognostic marker in colorectal cancer. Mod Pathol 19(10): 1317-1323.

Sepesi B, Watson TJ, Zhou D, Polomsky M, Litle VR, Jones CE, Raymond DP, Hu R, Qiu X, Peters JH (2010) Are endoscopic therapies appropriate for superficial submucosal esophageal adenocarcinoma? An analysis of esophagectomy specimens. J Am Coll Surg 210(4): 418-427.

Shabo I, Olsson H, Sun XF, Svanvik J (2009) Expression of the macrophage antigen CD163 in rectal cancer cells is associated with early local recurrence and reduced survival time. Int J Cancer 125(8): 1826-1831.

Shigeoka M, Urakawa N, Nakamura T, Nishio M, Watajima T, Kuroda D, Komori T, Kakeji Y, Semba S, Yokozaki H (2013) Tumor associated macrophage expressing CD204 is associated with tumor aggressiveness of esophageal squamous cell carcinoma. Cancer Sci 104(8): 1112-1119.

Sica A, Mantovani A (2012) Macrophage plasticity and polarization: in vivo veritas. J Clin Invest 122(3): 787-795.

Steevens J, Botterweck AA, Dirx MJ, van den Brandt PA, Schouten LJ (2010) Trends in incidence of oesophageal and stomach cancer subtypes in Europe. Eur J Gastroenterol Hepatol 22(6): 669-678.

Stewart DA, Yang Y, Makowski L, Troester MA (2012) Basal-like breast cancer cells induce phenotypic and genomic changes in macrophages. Mol Cancer Res 10(6): 727-738.

Stiles BM, Mirza F, Coppolino A, Port JL, Lee PC, Paul S, Altorki NK (2011) Clinical T2-T3N0M0 esophageal cancer: the risk of node positive disease. Ann Thorac Surg 92(2): 491-496; discussion 496-498.

Wang Q, Ni H, Lan L, Wei X, Xiang R, Wang Y (2010) Fra-1 protooncogene regulates IL- 6 expression in macrophages and promotes the generation of M2d macrophages. Cell Res 20(6): 701-712.

Ward SG, Mrsny R (2009) New insights into mechanisms of gastrointestinal inflammation and cancer. Curr Opin Pharmacol 9(6): 677-679.

Wijnhoven BP, Tran KT, Esterman A, Watson DI, Tilanus HW (2007) An evaluation of prognostic factors and tumor staging of resected carcinoma of the esophagus. Ann Surg 245(5): 717-725.

Zhang JQ, Hooker CM, Brock MV, Shin J, Lee S, How R, Franco N, Prevas H, Hulbert A, Yang SC (2012) Neoadjuvant chemoradiation therapy is beneficial for clinical stage T2 N0 esophageal cancer patients due to inaccurate preoperative staging. Ann Thorac Surg 93(2): 429-435; discussion 436-427.

This work is published under the standard license to publish agreement. After 12 months the work will become freely available and the license terms will switch to a Creative Commons AttributionNonCommercial-Share Alike 4.0 Unported License.

\section{Supplementary Information accompanies this paper on British Journal of Cancer website (http://www.nature.com/bjc)}

